\title{
ON THE COMPETITION BETWEEN RADIAL EXPANSION AND COULOMB COLLISIONS IN SHAPING THE ELECTRON VELOCITY DISTRIBUTION FUNCTION: KINETIC SIMULATIONS
}

\author{
S. LANDi ${ }^{1}$, L. Matteini ${ }^{1}$, And F. Pantellini ${ }^{2}$ \\ ${ }^{1}$ Dipartimento di Fisica e Astronomia, Università degli Studi di Firenze Largo E. Fermi 2, I-50125 Firenze, Italy \\ 2 LESIA, Observatoire de Paris 5, Place J. Janssen, F-92195 Meudon Cedex, France \\ Received 2012 August 31; accepted 2012 October 12; published 2012 November 16
}

\begin{abstract}
We present numerical simulations of the solar wind using a fully kinetic model which takes into account the effects of particle's binary collisions in a quasi-neutral plasma in spherical expansion. Starting from an isotropic Maxwellian velocity distribution function for the electrons, we show that the combined effect of expansion and Coulomb collisions leads to the formation of two populations: a collision-dominated cold and dense population almost isotropic in velocity space and a weakly collisional, tenuous field-aligned and antisunward drifting population generated by mirror force focusing in the radially decreasing magnetic field. The relative weights and drift velocities for the two populations observed in our simulations are in excellent agreement with the relative weights and drift velocities for both core and strahl populations observed in the real solar wind. The radial evolution of the main moments of the electron velocity distribution function is in the range observed in the solar wind. The electron temperature anisotropy with respect to the magnetic field direction is found to be related to the ratio between the collisional time and the solar wind expansion time. Even though collisions are found to shape the electron velocity distributions and regulate the properties of the strahl, it is found that the heat flux is conveniently described by a collisionless model where a fraction of the electron thermal energy is advected at the solar wind speed. This reinforces the currently largely admitted fact that collisions in the solar wind are clearly insufficient to force the electron heat flux obey the classical Spitzer-Härm expression where heat flux and temperature gradient are proportional to each other. The presented results show that the electron dynamics in the solar wind cannot be understood without considering the role of collisions.
\end{abstract}

Key words: methods: numerical - plasmas - solar wind

Online-only material: color figures

\section{INTRODUCTION}

The solar wind is a low density, weakly collisional plasma. The electron mean free path at $1 \mathrm{AU}$ is of the order of $10^{8} \mathrm{~km}$, comparable to the characteristic large scale variations of macroscopic quantities such as density, temperature, and pressure. Under such circumstances, it is not surprising that the electron velocity distribution function (eVDF) is far from the Maxwell-Boltzmann distribution function of a gas at thermodynamical equilibrium. The typical eVDF observed in the solar wind is mostly the superposition of a quasi-isotropic "core," an extended "halo" at energies beyond about $100 \mathrm{eV}$, and a narrow "strahl" aligned with the magnetic field and displaced in the antisunward direction with respect to the core at energies larger than about $50 \mathrm{eV}$ (Feldman et al. 1975; Rosenbauer et al. 1977; Pilipp et al. 1987).

The characteristics of the eVDF have been modeled in different ways over the years. For example, Feldman et al. (1975, 1978a) and Pilipp et al. (1987) described the eVDF using essentially a drifting (with respect the bulk speed) bi-Maxwellian distribution function for the low-energy part of the eVDF (the "core") and another drifting bi-Maxwellian component for the high-energy tails (the "hot" or the "halo" component). A different approach has been followed by Maksimovic et al. (1997b) who used Lorentzian (or Kappa) distribution functions to fit the three-dimensional eVDF measured with the Ulysses spacecraft, averaged over all directions. Maksimovic et al. (2005) reduced the eVDF to a non-drifting bi-Maxwellian for the core, a nondrifting Kappa distribution function for the halo, and the "strahl" being defined as the residual population in the observed distribution. In a similar way, Štverák et al. (2008) analyzed several properties of the eVDF obtained by instruments on board Helios, Cluster, and Ulysses. More recently, Štverák et al. (2009) added to the model used by Maksimovic et al. (2005) and Štverák et al. (2008) a truncated and drifting Lorentzian distribution to better represent the observed macroscopic characteristics of the "strahl" component. In a similar way, Feldman et al. (1982) and Phillips \& Gosling (1990) used a Lorentzian distribution for the halo, a model for the strahl, and modeled the residual part (the cold or core component) using a bi-Maxwellian distribution function.

Several attempts have been made to asses the radial electron temperature gradient. The logarithmic slopes of the total electron temperature $\beta_{\mathrm{e}}$ as well as the power-law indices of the core and halo populations, $\beta_{\mathrm{c}}$ and $\beta_{\mathrm{h}}$, respectively, have been estimated in the past using several instruments and methods, giving rise to a large spread in their estimations: while Montgomery et al. (1968), using Vela 4 data, found $\beta_{\mathrm{e}}$ in the range $[-0.4,-0.2]$, subsequent measurements with Interplanetary Monitoring Platform (IMP; Feldman et al. 1978b), Helios (Rosenbauer et al. 1977; Marsch et al. 1989; Pilipp et al. 1990), and Ulysses spacecrafts (Maksimovic et al. 2000), found that the radial gradient was generally steeper than $\beta_{\mathrm{e}}=-2 / 7$ predicted for a collisional heat flux dominated model (Hartle \& Sturrock 1968), but flatter than the adiabatic value $\beta_{\mathrm{e}}=-4 / 3$. Helios data showed slopes in the range $[-0.7,-0.25]$ (Marsch et al. 1989; Pilipp et al. 1990) depending on the kind of solar wind considered with a less steep gradient for fast streams. On the contrary, out-of-ecliptic Ulysses data during solar minimum gave slopes around $[-0.9,-0.8]$ (Maksimovic et al. 2000). Combining Helios and Ulysses data, Maksimovic et al. (2005) showed that the electron gradient power-law index can be in the 
range $[-0.9,-0.6]$. Also, the core electron temperature gradient shows a large spread estimation. Slopes of about $\beta_{\mathrm{c}}=-0.35$ were found using Mariner 10 and Voyager 2 data (Ogilvie \& Scudder 1978; Sittler \& Scudder 1980; Sittler et al. 1981). A steeper gradient with slopes around $[-0.9,-0.6]$ has been obtained using IMP and Mariner 10 (Feldman et al. 1979), Helios (Pilipp et al. 1990), and Ulysses (Scime et al. 1994; Phillips et al. 1995a; Issautier et al. 1998; Maksimovic et al. 2000) data. For the halo population, observations typically showed flatter gradients than the core with power-law index $\beta_{\mathrm{h}}$ in the range [-0.45, -0.25] (Scime et al. 1994; Phillips et al. 1995b; Maksimovic et al. 2000).

The solar wind electron heat flux is an important ingredient in the regulation of the expansion of the solar corona. Early measurements (e.g., Montgomery et al. 1968) found a qualitative agreement between the measured and the classical Spitzer-Härm collision-dominated electron heat flux (Spitzer \& Härm 1953). On the other hand, more recent observations at 1 AU using IMP 6, 7, and 8 spacecraft data (Feldman et al. 1975, 1976a, 1976b) and at 0.3 to 1 AU using Helios 1 and 2 data (Pilipp et al. 1987, 1990) indicate strong departure from the Spitzer-Härm value. These studies also show that in unperturbed fast streams, the heat flux is mainly carried by the high-energy field aligned electron population (e.g., Feldman et al. 1975). A more extensive study of the electron heat flux has been reported by Salem et al. (2003), who show that there is no clear correlation between heat flux strength and solar wind speed at $1 \mathrm{AU}$, whereas a correlation between the heat flux strength and the so-called collisional age could be established. Salem et al. (2003) also found that the Spitzer-Härm heat flux is an upper limit for the measured electron heat flux at $1 \mathrm{AU}$; this is probably a coincidence rather than the consequence of some sort of regulating mechanism. Scime et al. (1994) have analyzed the radial gradients of the electron heat flux from 1 to $5 \mathrm{AU}$ using the three-dimensional spectrometer on Ulysses. They found that the radial decrease can be expressed in terms of a power law with exponent $\gamma=-2.8$ for the global data set and a somewhat steeper gradient, $\gamma=-3.0$, when averaging over a solar rotation. The radial decrease of the electron heat flux is consistent with the local deposition of heat required to compensate for the non-adiabatic cooling of the electrons. The observed electron heat flux cannot be expressed in terms of the classical Spitzer-Härm expression even within the frame of a two-fluid model (electrons and protons; Cuperman et al. 1988). By fitting the electron velocity distributions observed in the solar wind with a bi-Maxwellian (core + drifting halo) velocity distribution function, Feldman et al. (1975) show that the electron heat flux is mainly carried by the halo population. The radial trend was observed to be consistent with a theoretical interpretation where the whistler heat flux instability reduces the halo-core relative drift velocity for solar wind conditions above the instability threshold (Gary et al. 1994). Scime et al. (1999) have shown that in the high latitude solar wind observed by Ulysses the heat flux component parallel to the magnetic field decreases as $r^{-2.9}$ with no clear correlation with the heliographic latitude or the solar wind speed. Similar results have been reported by Scime et al. (2001) using long term measurements of the electron heat flux from Ulysses.

From a theoretical point of view, the evolution of the eVDF, and its role in accelerating the solar wind, has been investigated using the collisionless (exospheric) approach. The earliest attempts (Jockers 1970; Lemaire \& Scherer 1971) were based on the assumption of isotropic Maxwellians while more recent works (Maksimovic et al. 1997a; Lamy et al. 2003; Zouganelis et al. 2004) treated the case of non-thermal distributions with an excess of high-energy particles. The consequences of neglecting collisions in exospheric models have been investigated by Zouganelis et al. (2005) by comparing exospheric calculations with kinetic simulations, including Coulomb-like collisions (e.g., Landi \& Pantellini 2003). The bottom line of the comparison is that even though electron collisions are rare in the solar wind, it appears that they play a role in fine shaping the $\mathrm{eVDF}$ and, as consequence, in regulating the radial evolution of macroscopic quantities.

The main objective of the present work is to investigate the effect of electron-electron and electron-proton collisions in shaping the eVDF in a radially supersonic expanding plasma. Despite the Knudsen number (the ratio between the mean-free path and the gradient scales of macroscopic quantities like the density or the temperature) in the supersonic solar wind being of the order of unity for both electrons and protons, the latter are much less affected by collisions than the former. The reason is that the solar wind is supersonic for protons and subsonic for electrons. Under such circumstances the relative position between protons is dominated by the expansion velocity which systematically increases the inter-particle distance, rather than by the thermal motion which has no systematic effect on the relative position between particles. Indeed, correlations between the shape of the eVDF and the ratio between the collisional time and expansion time, the so-called collisional age, have been shown to exist in the solar wind (Salem et al. 2003; Štverák et al. 2008).

Previous works have already discussed the effect of collisions on the properties of the solar wind electrons. Phillips \& Gosling (1990) have analyzed, within a fluid framework, the effect of Coulomb collisions in regulating the temperature anisotropy, showing that Coulomb collision can account for the temperature anisotropy observed at 1 AU. Using a test-particle approach, Lie-Svendsen et al. (1997) and Lie-Svendsen \& Leer (2000) were able to reproduce the high-energy tails observed in the eVDF. In their model, however, the macroscopic characteristics of the background plasma were computed from fluid models. A similar approach, where the background plasma is now obtained from exospheric models, has been used by Pierrard et al. (1999) to study the formation of high-energy suprathermal tails in the eVDF, as observed at $1 \mathrm{AU}$ concluding that, in absence of waves, Coulomb collisions and external forces are unable to account for the formation of these tails which must be present in the corona. With the same model, Pierrard et al. (2001b) studied the eVDF evolution between 2 and 14 solar radii, a region where the Knudsen number is significantly less than unity. More recently, an analogous approach, using a linearized Fokker-Planck operator, has been used by Smith et al. (2012) to follow the eVDF evolution in the acceleration region of the fast wind: although the region is characterized by a Knudsen number substantially less than unity, they observe a discrepancy between the computed heat flux and the classical Spitzer-Härm formula which is consistent with what already observed by Landi \& Pantellini (2003) with the kinetic approach used also in the present paper.

The work we present in this paper follows a fully kinetic approach using a model already used to study the heat flux properties in the low solar corona and the acceleration properties of a supersonic wind driven only by collisions and the neutralizing electric field (Landi \& Pantellini 2001, 2003). The model is essentially stationary and neglects wave particle 
interactions, excluding the effects of plasma heating through wave energy dissipation. The model can nevertheless provide clues on how the radial expansion and collisions both shape the eVDF and the macroscopic fluid quantities throughout the heliosphere. Mainly because of the non-inclusion of the effect of waves in the model, but also because of numerical constraints, we restrict our investigation to the interval from 0.3 to either 3 or $6 \mathrm{AU}$ where the solar wind has already reached its supersonic cruise velocity. The paper is organized as follows: in Sections 2 and 3 , we give details on the numerical model and the setup conditions of the simulations. The simulation results are presented in Section 4, and a discussion and conclusion are given in Section 5.

\section{THE MODEL}

Details of the model have been given elsewhere (Pantellini 2000; Pantellini \& Landi 2001; Landi \& Pantellini 2001, 2003) and will not be repeated here in full extent. We briefly recall the basic features. The model is based on the numerical integration of the one-dimensional motion of an equal number $N$ of electrons and protons allowed to move freely in the domain $r_{0}<r<r_{\max }$. Particles interact with the central gravitational field produced by a star of mass $M$ and the charge neutralizing electric field $\mathbf{E}=E(r) \hat{r}$, with $\hat{r}=\mathbf{r} / r$. The system, including the particles, is spherically symmetric so that the equations of motion for a particle of mass $m$ and charge $q$ are simply

$$
\begin{gathered}
\frac{d^{2} r}{d t^{2}}=-\frac{G M}{r^{2}}+\frac{L^{2}}{m^{2} r^{3}}+\frac{q}{m} E(r), \\
L \equiv m r v_{\perp}=\text { constant },
\end{gathered}
$$

where $G$ is the gravitational constant and $L$ is the magnitude of the angular momentum expressed in terms of the perpendicular velocity $v_{\perp}$ of the particle. According to the above equations, particles may be interpreted as thin spherical shells, centered on $r=0$, rather than as point particles. In Equations (1) and (2), the magnetic field does not appear explicitly. However, assuming a radial magnetic field (a rough approximation of the Sun's polar magnetic field)

$$
\mathbf{B}=B_{0}\left(\frac{r_{0}}{r}\right)^{2} \hat{r}
$$

no additional term has to be added in Equation (1) and Equation (2) is equivalent to the conservation of the magnetic moment $\mu \equiv m v_{\perp}^{2} / 2 B$, viz.,

$$
\frac{d}{d t} \frac{m v_{\perp}^{2}}{2 B}=\frac{1}{2 m B_{0} r_{0}^{2}} \frac{d L^{2}}{d t} .
$$

The model must be regarded as pertinent for the high latitude solar wind where the effects of the Sun's rotation are negligible and where the magnetic field lines are essentially radial. The charge neutralizing electric field in Equation (1) is obtained in a way similar to Landi \& Pantellini (2001). The profile is adjusted iteratively during the initialization phase until local charge neutrality and zero current is obtained in all points of the system. Collisions are introduced in the system according the following rules. Two particles with relative absolute velocity $u$ that happen to be simultaneously located at the same radial distance $r$ perform an elastic collision with a probability $P_{\alpha \beta}(u, r)=$ $R_{\alpha \beta}(u) r^{-2}$ where

$$
R_{\alpha \beta}(u)= \begin{cases}1 & \text { if } u<u_{\alpha \beta} \\ \left(u_{\alpha \beta} / u\right)^{4} & \text { otherwise }\end{cases}
$$

where the indices $\alpha$ and $\beta$ refer to the species of the colliding particles (electron-electron, electron-proton, and proton-proton). Thus, each time two particles meet, a random number $p$ in the range $[0,1]$ is drawn. If $p \leqslant R_{\alpha \beta}$ a collision is performed, while if $p>R_{\alpha \beta}$ particles simply ignore each other. According to Equation (5), a collision is systematically performed for any two particles of species $\alpha$ and $\beta$ meeting with relative velocity less than $u_{\alpha \beta}$. For larger velocities, the $u^{-4}$ dependence of the collision probability mimics the velocity dependence of the scattering cross section for Coulomb collisions, whereas the $r^{-2} \mathrm{de}$ pendence accounts for the spherical geometry of the problem. In the event of a collision, conservation of energy and momentum do not suffice to specify the orientation of the post collision velocities in the center-of-mass frame. In a fully three-dimensional system, all directions are equivalent and the orientation of the post collision velocities should be drawn uniformly over all possible directions. In our one-dimensional model, the collision frequency is highest for relative velocities along the radial direction and is essentially zero for relative velocities perpendicular to the radial direction. We therefore choose the angle $\theta$ between the radial direction and the post collision velocities of the particles in the center-of-mass frame according to $\theta=\arccos \sqrt{p}$, where $p$ is a random number uniformly distributed in the range $[0,1]$. With this particular choice, one ensures that the velocity distributions are isotropic in the strongly collisional limit (see chapter II.C in Pantellini 2000). Pantellini \& Landi (2001) and Landi \& Pantellini (2001) have shown that using cutoff velocities $u_{\alpha \beta}$ of the order of, or smaller than, the typical relative velocity between particles of species $\alpha$ and $\beta$, the transport properties of such a plasma are very much the same as those of a Fokker-Planck plasma.

The treatment of particles reaching one of the two boundaries in the system is as follows. Each time a particle reaches the lower boundary $r=r_{0}$ it is re-injected back into the system at $r=r_{0}$, according to a specified velocity distribution function, one for each species. At the upper boundary $r=r_{\max }$, protons are supersonic and almost all of them will be re-injected at the lower boundary $r=r_{0}$. Only a small fraction of protons will be injected back into the system corresponding to the negative velocity part of the prescribed proton velocity distribution function at $r=r_{\max }$. On the contrary, electrons are subsonic and a significant fraction of them must be re-injected back into the system from the top at $r=r_{\max }$, the choice of whether a given electron will be injected at the top or at the bottom being determined by the equal proton and electron flux (zero current) condition imposed at $r=r_{\max }$. Since the total number of particles in the system is kept constant, this automatically ensures zero current everywhere in the system once the stationary regime has been reached. The velocity distribution function for the electrons injected from the upper boundary is a drifting bi-Maxwellian having the same parallel and perpendicular temperatures as that of the outgoing electrons and same bulk velocity as protons. Distribution functions and their moments shown in this paper have been obtained once a stationary state with equal proton and electron densities in all parts of the system has been reached.

\section{SIMULATION SETUP}

We assume to be located sufficiently far from the stellar surface that the gravitational force in Equation (1) can be neglected. The velocity distributions prescribed at the inner boundary are characterized, for both electrons and protons, by 
Table 1

List of Parameter Used in the Simulations

\begin{tabular}{lccr}
\hline \hline$N$ & $\begin{array}{c}w_{0} \\
\left(\mathrm{~km} \mathrm{~s}^{-1}\right)\end{array}$ & $\begin{array}{c}L \\
(\mathrm{AU})\end{array}$ & $\begin{array}{c}n_{0} \\
\left(\mathrm{~cm}^{-3}\right)\end{array}$ \\
\hline 400 & 700 & 3 & 3 \\
1600 & 700 & 3 & 12 \\
3600 & 700 & 3 & 27 \\
6400 & 700 & 3 & 49 \\
400 & 350 & 3 & 3 \\
1600 & 350 & 3 & 13 \\
3600 & 350 & 3 & 27 \\
6400 & 350 & 3 & 50 \\
\hline 1600 & 700 & 6 & 6 \\
3600 & 700 & 6 & 13 \\
6400 & 700 & 6 & 23 \\
10,000 & 700 & 6 & 36 \\
1600 & 700 & 6 & 6 \\
3600 & 700 & 6 & 13 \\
6400 & 700 & 6 & 24 \\
10,000 & 700 & 6 & 38 \\
\hline
\end{tabular}

a drifting bi-Maxwellian

$$
f_{\alpha}=\left(\frac{m_{\alpha}}{2 \pi k_{\mathrm{B}}}\right)^{3 / 2} \frac{1}{T_{\alpha \perp} T_{\alpha \|}^{1 / 2}} e^{-\frac{m_{\alpha}}{2 k_{\mathrm{B}}}\left[\frac{\left(v_{\|}-w_{0}\right)^{2}}{T_{\alpha \|}}+\frac{v_{\perp}^{2}}{T_{\alpha \perp}}\right]}
$$

where $\alpha=\{e, p\}$ designs the species, $m_{\alpha}$ the mass, $k_{\mathrm{B}}$ is the Boltzmann constant, and $T_{\alpha \|}, T_{\alpha \perp}$ the temperatures parallel and perpendicular to the radial direction, respectively. Note that the drift velocity $w_{0}$ is the same for both species. We consider typical values for fast streams in the inner heliosphere beyond $0.3 \mathrm{AU}$, with a mean velocity $w_{0}=700 \mathrm{~km} \mathrm{~s}^{-1}$. For the protons, we assume a mean temperature $T_{p}=\left(T_{p \|}+2 T_{p \perp}\right) / 3=$ $3 \times 10^{5} \mathrm{~K}$ (Schwenn 1990) with a temperature anisotropy $A_{p}=T_{p \perp} / T_{p \|}=2$ (Matteini et al. 2007). For the electrons, we choose a mean temperature $T_{e}=\left(T_{e \|}+2 T_{e \perp}\right) / 3=1.5 \times 10^{5} \mathrm{~K}$. Since one goal of the present work is to study the radial evolution of the electron anisotropy, we begin the simulations with an isotropic eVDF, i.e., $A_{e}=T_{e \perp} / T_{e \|}=1.0$.

For the given values of the electron and proton temperatures and a typical density $n \sim 10 \mathrm{~cm}^{-3}$, the Fokker-Planck electron collision frequency is about $v_{e} \sim 4 \times 10^{-5} \mathrm{~s}^{-1}$, corresponding to an electron mean-free path of the order of $0.3 \mathrm{AU}$. Assuming a system length $L=r_{\max }-r_{0}=3 \mathrm{AU}$, the same collision frequency is obtained in the simulation model for a number of electrons $N \simeq 1000$ and a cutoff velocity for the electron-electron collisions $u_{e e}=0.4 v_{e e}$, where $v_{e e}^{2}=2 v_{e}^{2}=4 k_{B} T_{e} / m_{e}$ is the typical relative velocity between colliding particles at $r=r_{0}$ (Landi \& Pantellini 2001). The electron-proton and proton-proton cutoff velocities are scaled so that the ratio between the cutoff velocity and the most probable relative speeds between colliding particles is the same, i.e., $u_{\alpha \beta}=0.4 v_{\alpha \beta}$.

In the simulations and in the paper, unless differently specified, temperatures are expressed in terms of $T_{0} \equiv T_{\mathrm{e} \|}$, velocities in terms of $v_{0}=\sqrt{2 k_{\mathrm{B}} T_{0} / m_{e}}$, and distances in terms of $r_{0}$. With these units a bulk speed of $700 \mathrm{~km} \mathrm{~s}^{-1}$ corresponds to $w_{0}=0.3 v_{0}$. Given our objective to investigate the effect of Coulomb collisions in regulating the eVDF against the spherical expansion, we have performed a set of simulations with different densities, i.e., different number of particles, $N$, and different expansion velocities, namely, $w_{0}=700$ and $350 \mathrm{~km} \mathrm{~s}^{-1}$, respectively. The simulations are listed in Table 1.

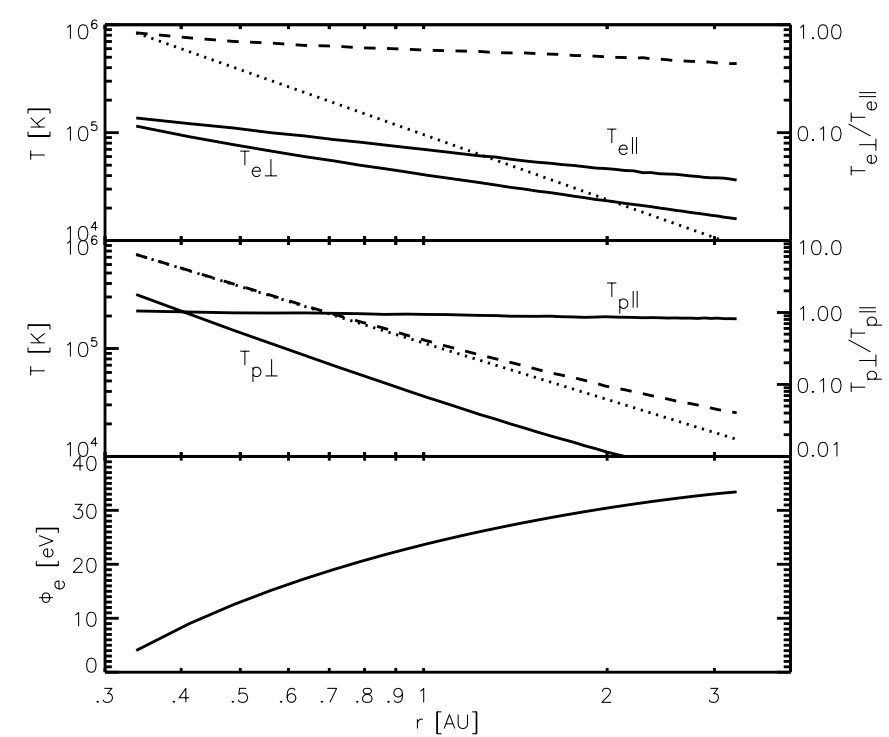

Figure 1. Some basic quantities as a function of the radial distance for a typical simulation (specifically for $n_{0}=27 \mathrm{~cm}^{-3}$ and $w_{0}=350 \mathrm{~km} \mathrm{~s}^{-1}$ ). Top panel: parallel and perpendicular electron temperature (solid), total electron temperature anisotropy $T_{\mathrm{e} \perp} / T_{\mathrm{e} \|}$ (dashed), and the double adiabatic prediction (dotted). Middle panel: same quantities for the proton temperature and temperature anisotropies. Bottom panel: electric potential energy for the electrons.

\section{RESULTS}

Typical radial profiles of the main macroscopic quantities are shown in Figure 1 for the case $n_{0}=27 \mathrm{~cm}^{-3}$ and $w_{0}=350 \mathrm{~km} \mathrm{~s}^{-1}$. For the protons, collisions do not seem to play a significant role in controlling the temperature anisotropy. The proton perpendicular temperature decreases very rapidly while the parallel temperature remains almost constant. The temperature anisotropy increases slightly slower than predicted by the adiabatic invariants in a radially expanding wind (Chew et al. 1956). On the contrary, for the electrons, collisions are observed to be important in regulating the thermal anisotropy which remains substantially smaller than the adiabatic prediction.

Although there is no gravity in the system, an electric field is needed to ensure equal densities and bulk velocities for electrons and protons. The electrostatic potential energy difference between the lower and the upper boundary is about $30 \mathrm{eV}$, which corresponds to about twice the thermal energy of the electrons at the lower boundary $(19 \mathrm{eV})$. The reason for the existence of an electric field is due to the different behavior of the subsonic electrons with respect to the supersonic protons in a spherical collisionless expansion. For an isotropic drifting Maxwellian distribution function at a given location, say $r_{0}$, and no collisions and electric field, the phase space density at larger distances is regulated by the combined effect of kinetic energy and magnetic moment conservation. Magnetic moment conservation implies that perpendicular velocity is constrained by $v_{\perp} \propto 1 / r$ (see Equation (2)) and thus $v_{\perp}$ must decrease with $r$. At the same time, the kinetic energy conservation forces the particle to move along a circle centered on zero velocity. Since the particle's velocity distribution function is centered around the bulk velocity, the expansion causes the flattening and the deformation of the initially circular velocity distribution function contours, as illustrated in Figure 2.

For the supersonic protons, the fact that the bulk velocity is much larger than the thermal velocity leads to the formation of a cigar-like distribution still compatible with a bi-Maxwellian description (upper right panel of Figure 2) and follows the 

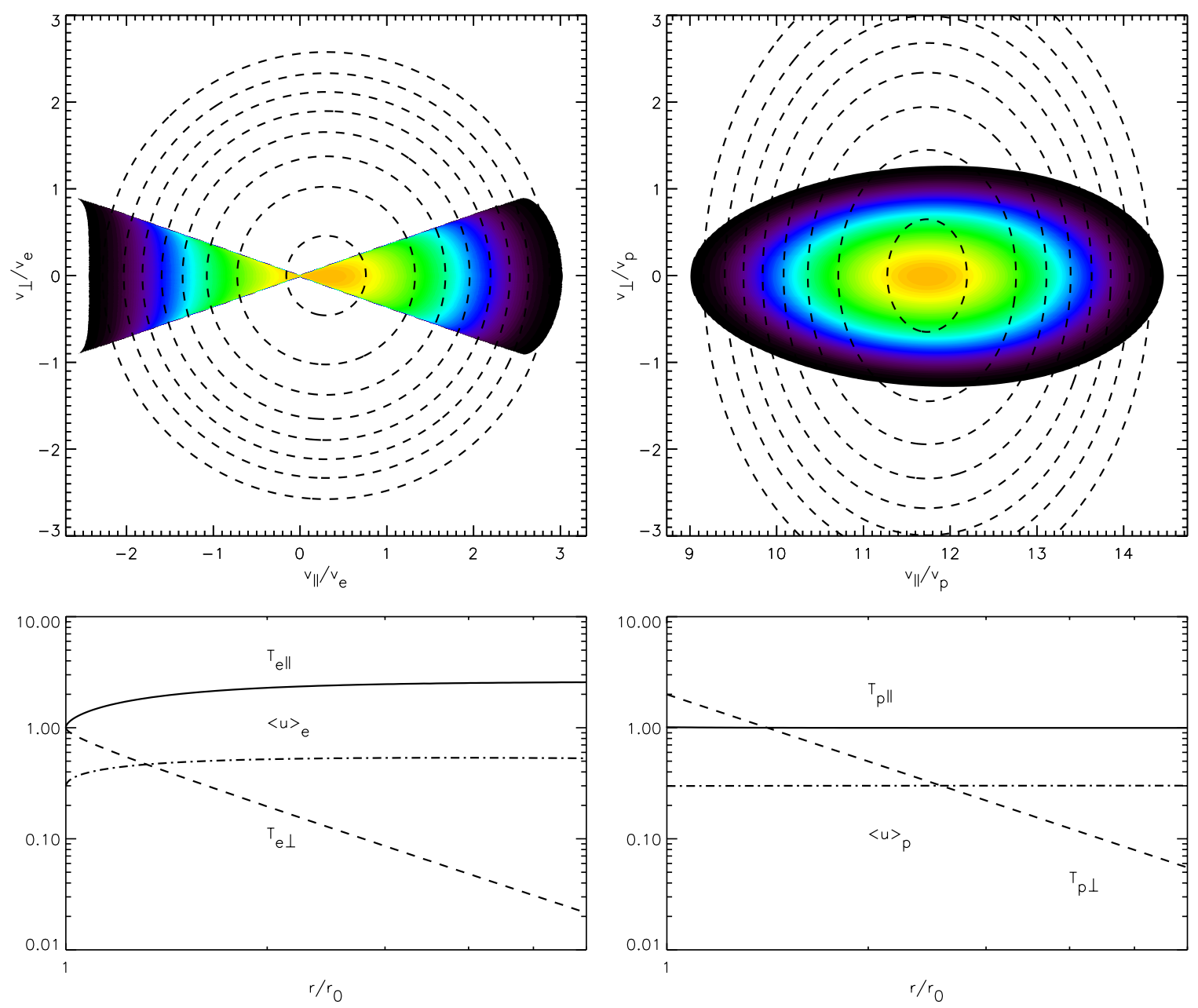

Figure 2. Top: velocity distribution function at $r=3 r_{0}$ obtained by applying Liouville's theorem, assuming a drifting distribution as given by Equation (6) at $r=r_{0}$ (dashed lines). Left and right panels are for a subsonic and supersonic population, respectively, corresponding to the parameters for protons and electrons at the base of the simulation domain. Bottom: spatial variation of the moments for the subsonic (left) and the supersonic (right) case, respectively.

(A color version of this figure is available in the online journal.)

prediction from double-adiabatic theory (Schulz \& Eviatar 1973) with constant parallel temperature and decreasing perpendicular temperature, as shown in the bottom right panel of Figure 2. For a subsonic population, like the electrons, the picture is completely different. The application of Liouville's theorem with energy and magnetic moment conservation leads to the formation of an asymmetric velocity distribution function (left top panel of Figure 2) where the perpendicular cooling is compensated by parallel heating and bulk flow acceleration (bottom left panel). As a consequence of the acceleration, an electric field is generated to prevent unequal proton and electron fluxes similar to what we observe in our simulations. Note that the electrostatic potential energy gain or loss across the system (see Figure 1) is of the order of the electron thermal energy only, i.e., much less than the mean kinetic energy of the highly supersonic protons. Under such circumstances, the zero field approximation underlying the simplistic model of Figure 2 is acceptable and its prediction concerning the radial evolution of the VDF pertinent, at least at the lowest level of details.

The two-dimensional structure of the eVDFs for four different case simulations is shown in Figures 3 and 4. In Figure 3, we report the eVDF in the slow stream case, $w_{0}=350 \mathrm{~km} \mathrm{~s}^{-1}$, for a low $\left(n_{0}=4 \mathrm{~cm}^{-3}\right)$ and a high $\left(n_{0}=75 \mathrm{~cm}^{-3}\right)$ density case. Distribution functions for the same densities in the fast stream case, $w_{0}=700 \mathrm{~km} \mathrm{~s}^{-1}$, are reported in Figure 4. All eVDFs are measured at $r=6 r_{0}$, corresponding to a heliocentric distance 1.8 AU in physical units. From the figure it is possible to recognize two different electron populations: a relatively isotropic cold core and an elongated and an anisotropic hot component. The core component is collision-dominated and its relative weight is regulated by the electron density, i.e., by the collision frequency. On the contrary, the hot population is nearly collisionless and its anisotropy is the effect of the focusing due to the angular momentum conservation as illustrated in Figure 2.

A quantitative analysis of the two population's characteristics can be performed by a fitting procedure with an analytical model of the form

$$
g_{e}=g_{\mathrm{c}}+g_{\mathrm{h}} .
$$

We consider that the cold (subscript "c") and hot (subscript "h") populations can be described as a sum of two bi-Maxwellians

$$
\begin{aligned}
& g_{e}=\left(\frac{m_{e}}{2 \pi k_{\mathrm{B}}}\right)^{3 / 2} \frac{n_{\mathrm{c}}}{T_{\mathrm{c} \perp} T_{\mathrm{c} \|}^{1 / 2}} e^{-\frac{m_{e}}{2 k_{\mathrm{B}}}\left[\frac{\left(v_{\|}-w_{\mathrm{c}}\right)^{2}}{T_{\mathrm{c} \|}}+\frac{v_{\perp}^{2}}{T_{\mathrm{c} \perp}}\right]} \\
& +\left(\frac{m_{e}}{2 \pi k_{\mathrm{B}}}\right)^{3 / 2} \frac{n_{\mathrm{h}}}{T_{\mathrm{h} \perp} T_{\mathrm{h} \|}^{1 / 2}} e^{-\frac{m_{e}}{2 k_{\mathrm{B}}}\left[\frac{\left(v_{\|}-w_{\mathrm{h}}\right)^{2}}{T_{\mathrm{h} \|}}+\frac{v_{\perp}^{2}}{T_{\mathrm{h} \perp}}\right]},
\end{aligned}
$$



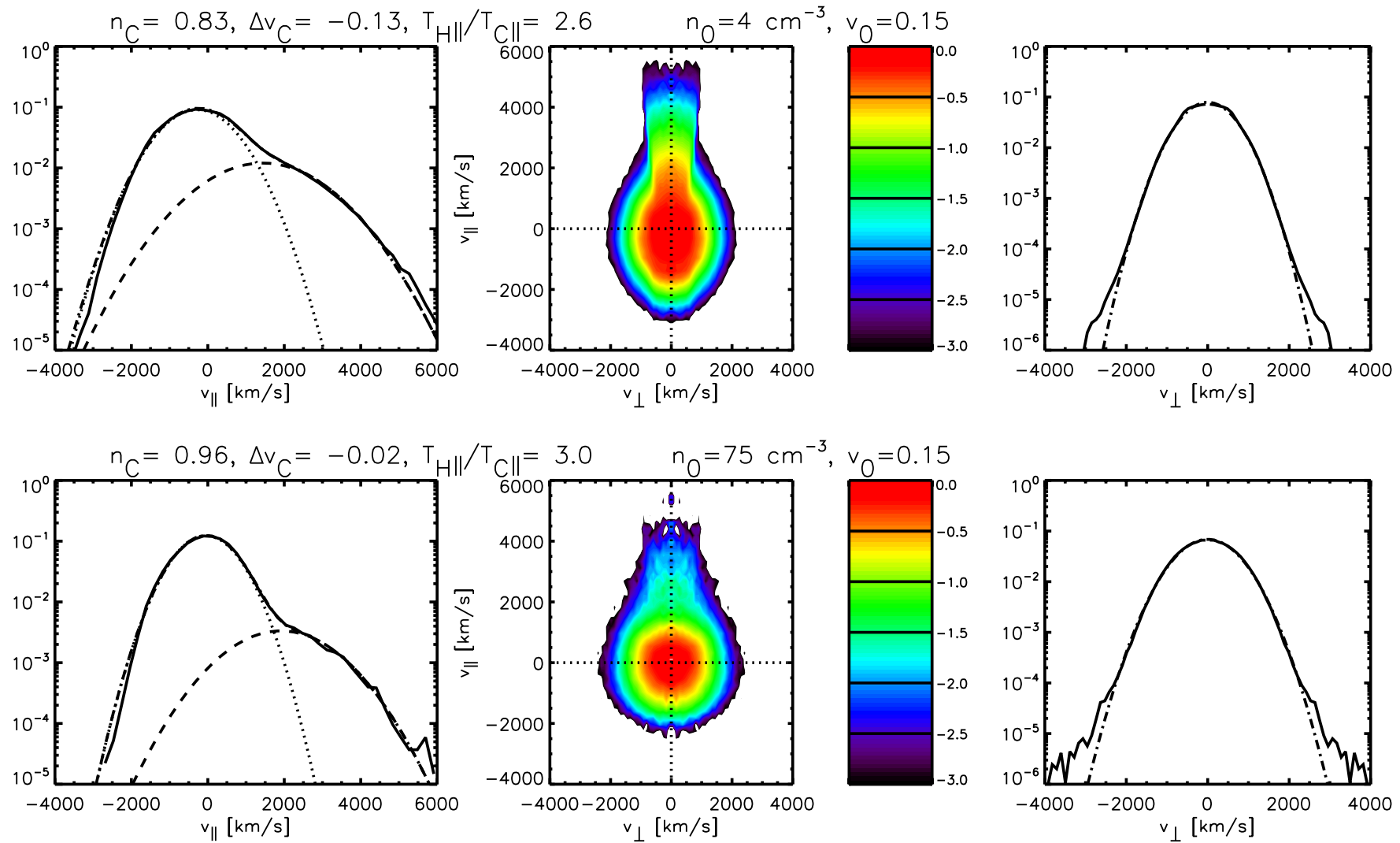

Figure 3. Electron velocity distribution functions at $r \simeq 1.8 \mathrm{AU}$ for two simulations with bulk velocity $w_{0}=350 \mathrm{~km} \mathrm{~s}^{-1}$. Top and bottom panels refer to a dilute and dense wind, respectively.

(A color version of this figure is available in the online journal.)
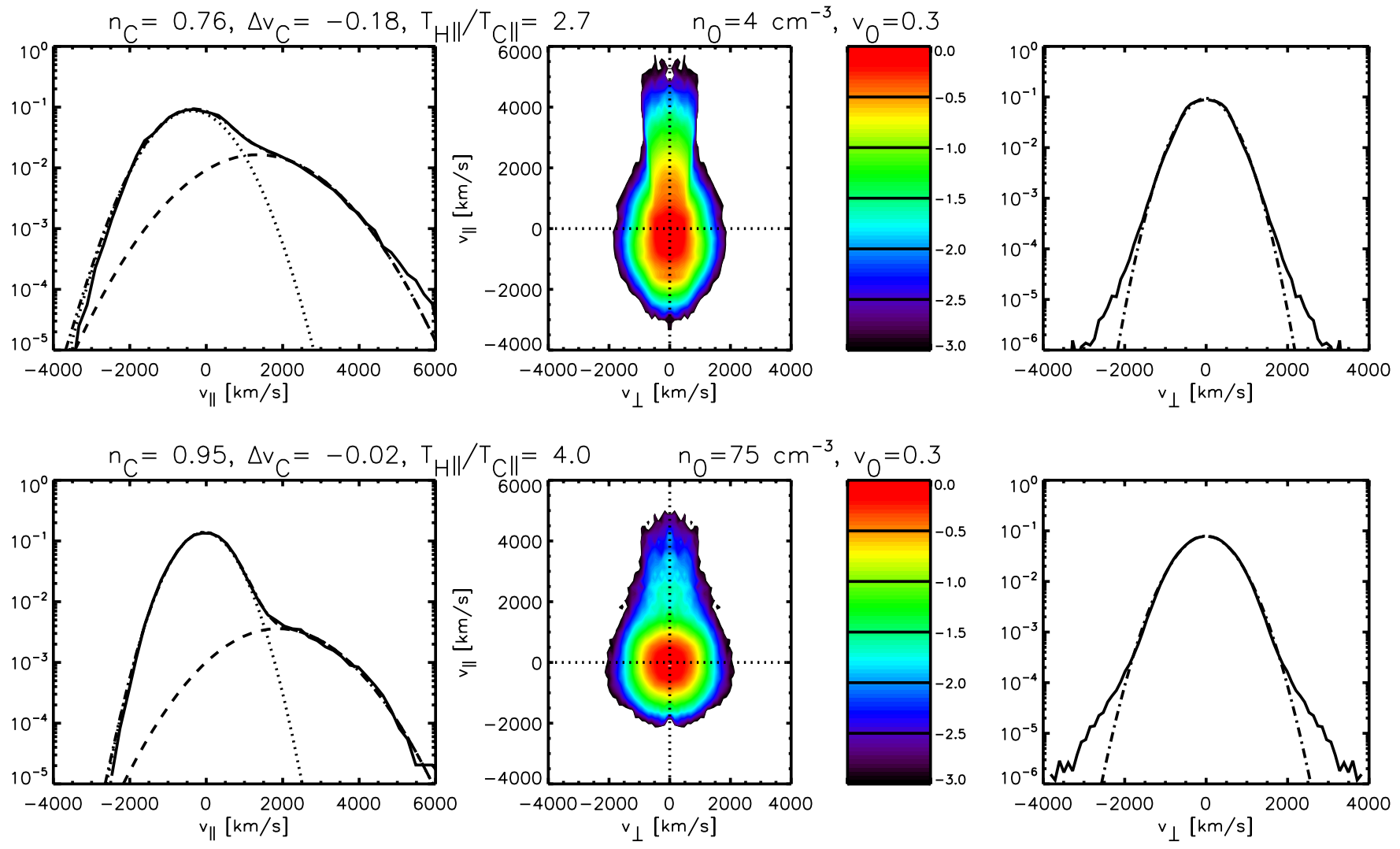

Figure 4. Electron velocity distribution functions at $r \simeq 1.8 \mathrm{AU}$ for two simulations with bulk velocity $w_{0}=700 \mathrm{~km} \mathrm{~s}^{-1}$. Top and bottom panels refer to a dilute and dense wind, respectively.

(A color version of this figure is available in the online journal.) 


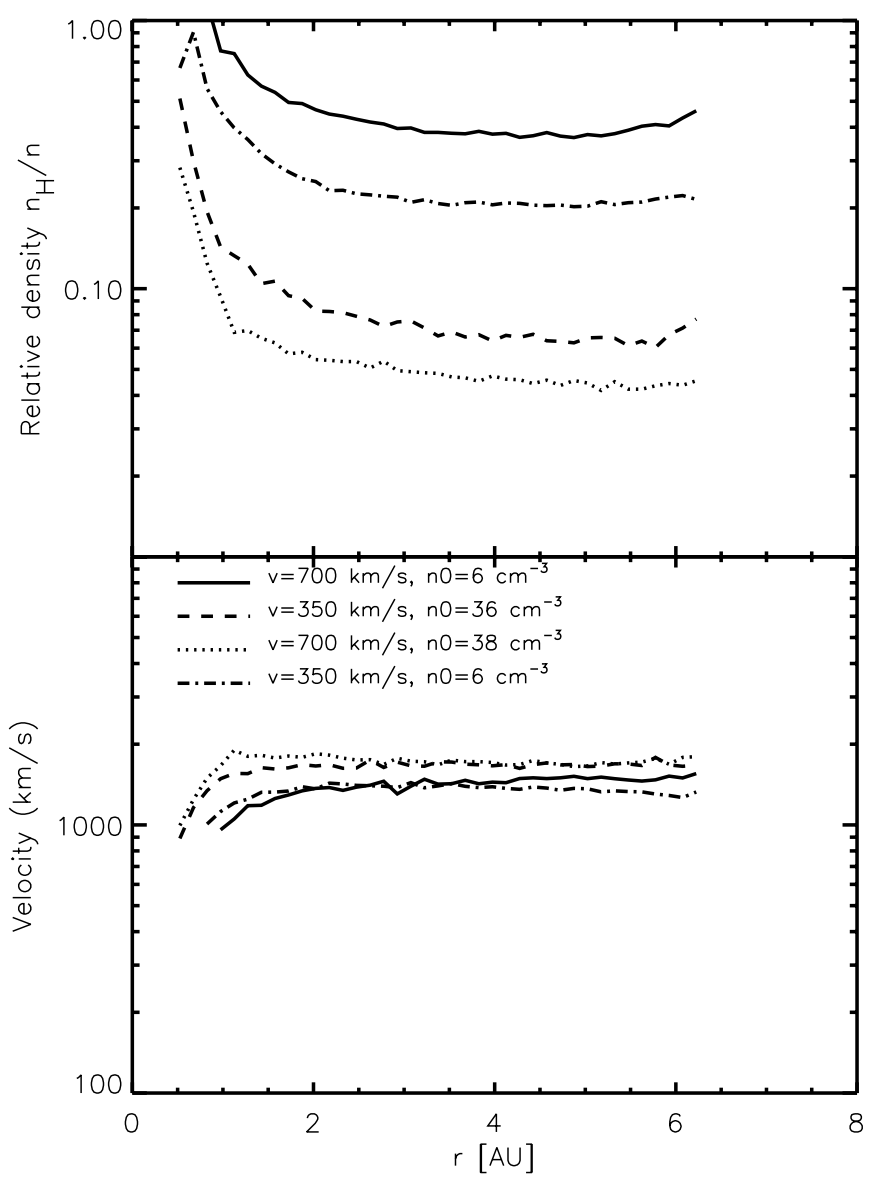

Figure 5. Top panel: electron halo density relative to the total electron density as function of the heliocentric distance. Bottom panel: drift velocities of the hot electron population with respect to the protons. Curves refer to simulations with different wind bulk speeds and densities as indicated.

where, $n_{\mathrm{c}}$ and $n_{\mathrm{h}}$ are the densities of the two populations and $w_{\mathrm{c}}$ and $w_{\mathrm{h}}$ their drift velocities with respect the bulk speed of the whole distribution. Otherwise, notations are the same as for Equation (6). Assuming a decomposition of the eVDF as given by Equation (8), in principle we have to fit eight parameters. However, parameters are constrained by the following relations:

$$
\begin{gathered}
n_{\mathrm{c}}+n_{\mathrm{h}}=n_{e} \\
n_{\mathrm{c}} w_{\mathrm{c}}+n_{\mathrm{h}} w_{\mathrm{h}}=0 \\
n_{\mathrm{c}} w_{\mathrm{c}}^{2}+n_{\mathrm{c}} k_{\mathrm{B}} T_{\mathrm{c} \|}+n_{\mathrm{h}} w_{\mathrm{h}}^{2}+n_{\mathrm{h}} k_{\mathrm{B}} T_{\mathrm{h} \|}=n_{e} m_{e}\left\langle v_{\|}^{2}\right\rangle_{e} \\
2 n_{\mathrm{c}} k_{\mathrm{B}} T_{\mathrm{c} \perp}+2 n_{\mathrm{h}} k_{\mathrm{B}} T_{\mathrm{h} \perp}=n_{e} m_{e}\left\langle v_{\perp}^{2}\right\rangle_{e},
\end{gathered}
$$

where $n_{e},\left\langle v_{\|}^{2}\right\rangle_{e}$, and $\left\langle v_{\perp}^{2}\right\rangle_{e}$ are the expected values for density and both the parallel and perpendicular kinetic energies computed from the observed eVDF. As the model distribution function depends nonlinearly on the fitting parameters, we adopt the iterative Levenberg-Marquardt fitting technique (Marquardt 1963) constrained by the relations (9)-(12).

\subsection{Density and Drift Velocities}

Figure 5 reports the relative densities of the hot population as a function of the heliospheric distance for four different simulations (top panel), as well as the relative drift velocities of the

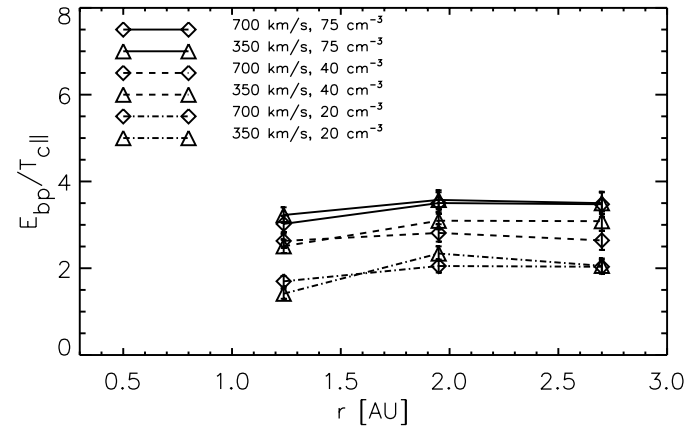

Figure 6. Break-point energy between cold and hot electron populations for various simulations.

hot population with respect to the protons (bottom panel). Apart from the most dilute cases, the relative density of the hot population is well beyond $10 \%$ of the total electron density. The drift velocities are between 1000 and $2000 \mathrm{~km} \mathrm{~s}^{-1}$. Both densities and drift velocities appear to be in good agreement with solar wind data at $1 \mathrm{AU}$ observed for the halo population (Feldman et al. 1978a; Pilipp et al. 1987, 1995b; here including the strahl population) and we do not need any anomalous frictional process to reduce the halo-core drift as suggested by Chen et al. (2003). In the simulations, the shaping of the eVDF can only be due to the combined action of collisions, expansion, and electric field. Scudder \& Olbert (1979) suggest that a transition between collisional and collisionless electron expansion occurs for a particle energy somewhat larger than $7 k_{\mathrm{B}} T_{\mathrm{c} \|}(r), T_{\mathrm{c} \|}(r)$ being the local parallel temperature of the cold population. In Figure 6, we observe that the break-point energy (corresponding to the point where the hot population starts dominating the cold population) is somewhere between 2 and $4 k_{\mathrm{B}} T_{\mathrm{c} \|}(r)$, clearly less than suggested by Scudder \& Olbert (1979). A clear correlation between wind density (i.e., collision frequency) and break-point energy value is also observed. Nevertheless, the break-point energy observed in our simulations appears to be compatible with observations of the break point for the so-called strahl population, i.e., the hot portion of the eVDF moving predominantly in the antisunward direction, form Helios, Cluster, and Ulysses measurements (Štverák et al. 2009). The discrepancy between our results and Scudder \& Olbert (1979) could be related to the fact that in the Scudder \& Olbert (1979) model the halo population is formed deep inside the corona, a region not included in our simulations.

\subsection{Temperatures}

The behavior of the mean electron temperature, defined as $\left(T_{e \|}+2 T_{e \|}\right) / 3$ as function of the radial distance, is shown in Figure 7 . All curves refer to fast stream simulations extending out to a heliocentric distance $L=6 \mathrm{AU}$. Base densities are $n_{0}=36,23$, and $13 \mathrm{~cm}^{-3}$ for the solid, dashed, and dot-dashed lines, respectively. Clearly, the temperature profile cannot be described by the adiabatic prediction $\propto r^{-4 / 3}$ or by a collisional heat flux dominated model $\propto r^{-2 / 7}$. This is not surprising. First, the presence of a beamed and hot population directed antisunward carries an important heat flux and, hence, the expansion cannot be an adiabatic one. Second, the $r^{-2 / 7}$ curve is based on the assumption of a Spitzer-Härm collisional flux, where small departures from the Maxwell-Boltzmann velocity distribution function and a collisional mean free path much smaller than the characteristic scales of variation of temperature and density are both assumed. These assumptions are also not 


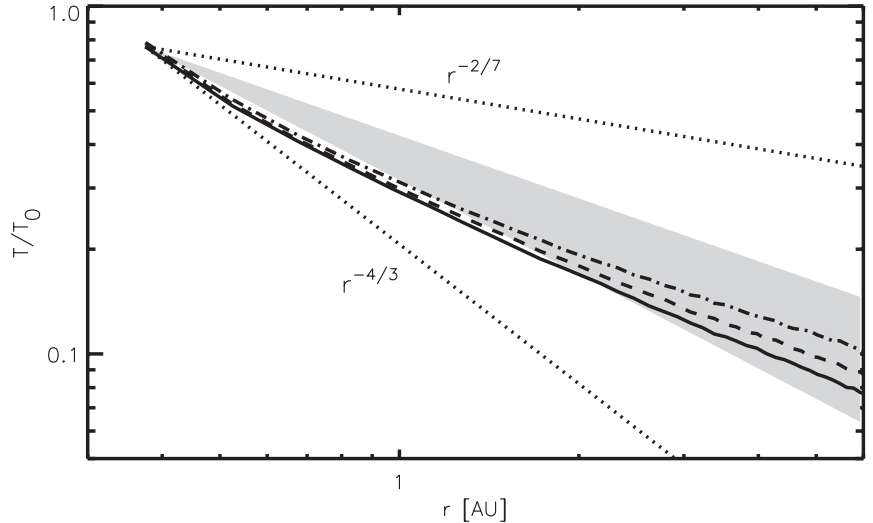

Figure 7. Mean electron temperature against radial distance for different simulations. All simulations refer to fast wind conditions with densities at the base varying between 36,23 , and $13 \mathrm{~cm}^{-3}$ (solid, dashed, and dot dashed curves). The $r^{-2 / 7}$ and $r^{-4 / 3}$ curves correspond to the collisional heat flux dominate case and the adiabatic case, respectively. The shaded region outlines the range of the electron temperature profiles from Ulysses measurements ranging from $T \propto r^{-0.6}$ to $T \propto r^{-0.9}$.

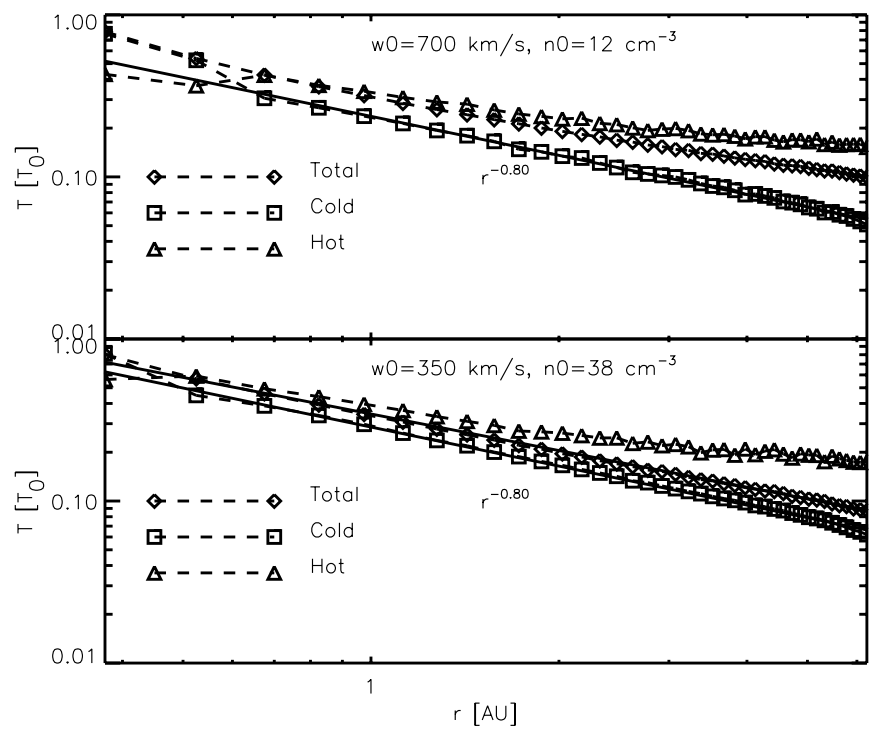

Figure 8. Mean electron temperature profiles for the cold, hot, and the whole electron population. Top and bottom panels refer to a tenuous fast wind and a dense slow wind, respectively.

met in our simulations. Again, the electron temperature profile appears to be in good agreement with Ulysses measurements of the total electron temperature with logarithmic slopes varying between $\beta_{\mathrm{e}}=-0.6$ and -0.9 (Maksimovic et al. 2000, 2005). However, the temperature profile in all the simulations do not have a constant logarithmic slope with a tendency to flatten at large distances. This is consistent with exospheric model predictions with a rather adiabatic behavior for the cold component and isothermal behavior of the hot component, the latter becoming more prominent with increasing heliocentric distance (Meyer-Vernet \& Issautier 1998). A flattening of the temperature profile with distance has also been inferred by Cranmer et al. (2009) combining electron data from Helios and Ulysses and over a smaller range by Le Chat et al. (2011) using the Quasi-Thermal Noise spectroscopy technique on Ulysses.

In Figure 8, are shown the cold, hot, and the total electron temperature profiles for two simulations: a tenuous fast wind and a dense slow wind, respectively. From the figure it is clear that the flattening of the total temperature is due to the hot
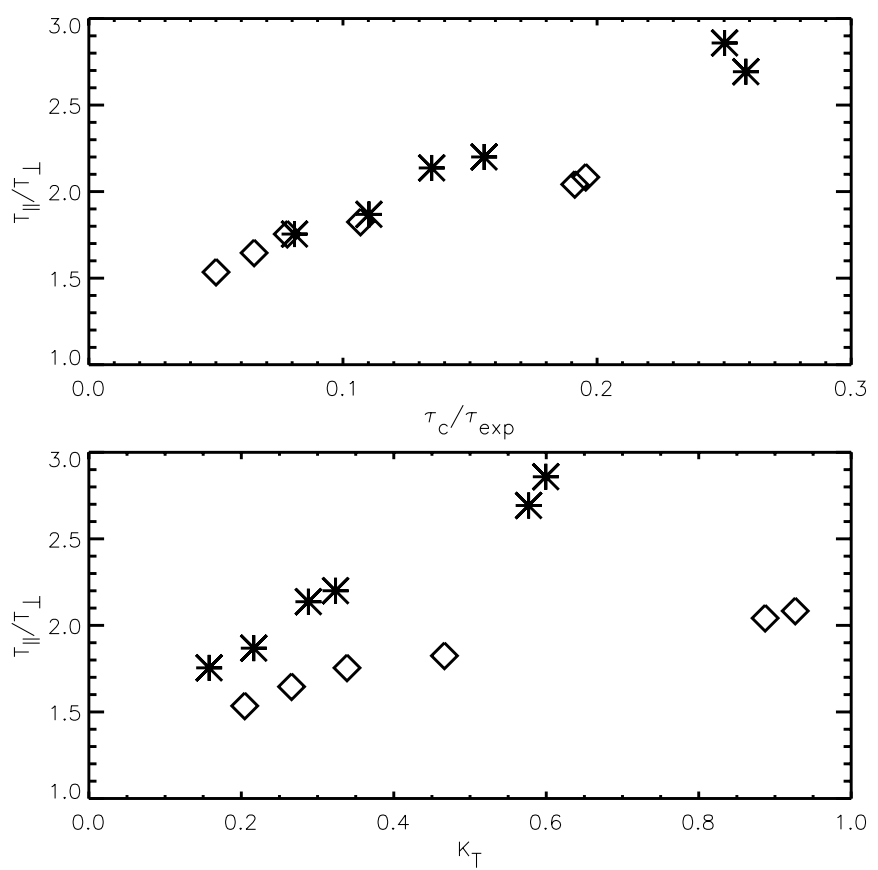

Figure 9. Total electron temperature anisotropy at $r=1.12 \mathrm{AU}$ as function of the collisional time to expansion time ratio (top panel) and of the Knudsen number (bottom panel) for all the simulations with $N>400$ listed in Table 1 . Asterisks refer to fast wind while square to slow wind simulations.

component. The cold component has a logarithmic slope of about -0.8 for both simulations, while the hot component has the tendency to follow an isothermal profile. The different total temperature evolution for the two simulations appears to be due to the different relative weight of the hot and cold components. As already stated, the isothermal temperature profile for the hot component is in agreement with the exospheric prediction (Meyer-Vernet \& Issautier 1998). This is not the case for the collisional-dominated cold component for which exospheric theory predicts an adiabatic temperature profile $\propto r^{-4 / 3}$, which we do not observe in our simulations. The discrepancy suggests the presence of a heat flux, obviously not measurable using a biMaxwellian fit for the cold core velocity distribution function. Moreover, the $-4 / 3$ prediction from Meyer-Vernet \& Issautier (1998) is based on the assumption of an isotropic velocity distribution function for the cold component, while, as we will see below, this is not entirely true in our simulations.

In Figures 9 and 10, the parallel to perpendicular temperature anisotropy values at a prescribed heliocentric distance, near $1 \mathrm{AU}$, are plotted. All simulations listed in Table 1 with $N>400$ are plotted, i.e., all simulations with a density larger than $1 \mathrm{~cm}^{-3}$ at 1 AU. Figure 9 shows the anisotropy considering the whole distribution function, while in Figure 10 only the cold component is considered. Higher values of the temperature anisotropy are obviously obtained for the whole distribution function. Anisotropies are in the range 1.5-3.0, in good agreement with observations by ISEE3 (Phillips et al. 1989), but somewhat higher than that reported by Salem et al. (2003) using WIND data. Cold component temperature anisotropies are somewhat smaller, covering the range 1.2-2, also in good agreement with core component measurements from Helios, Cluster, and Ulysses (Štverák et al. 2008). In the top panels of both Figures (9 and 10), temperature anisotropies are plotted against the collisional age, defined as the ratio between the collisional time $\tau_{\mathrm{c}}=1 / \nu_{\mathrm{ep}}$ and the expansion time $\tau_{\mathrm{exp}}=L / v_{0}$, 

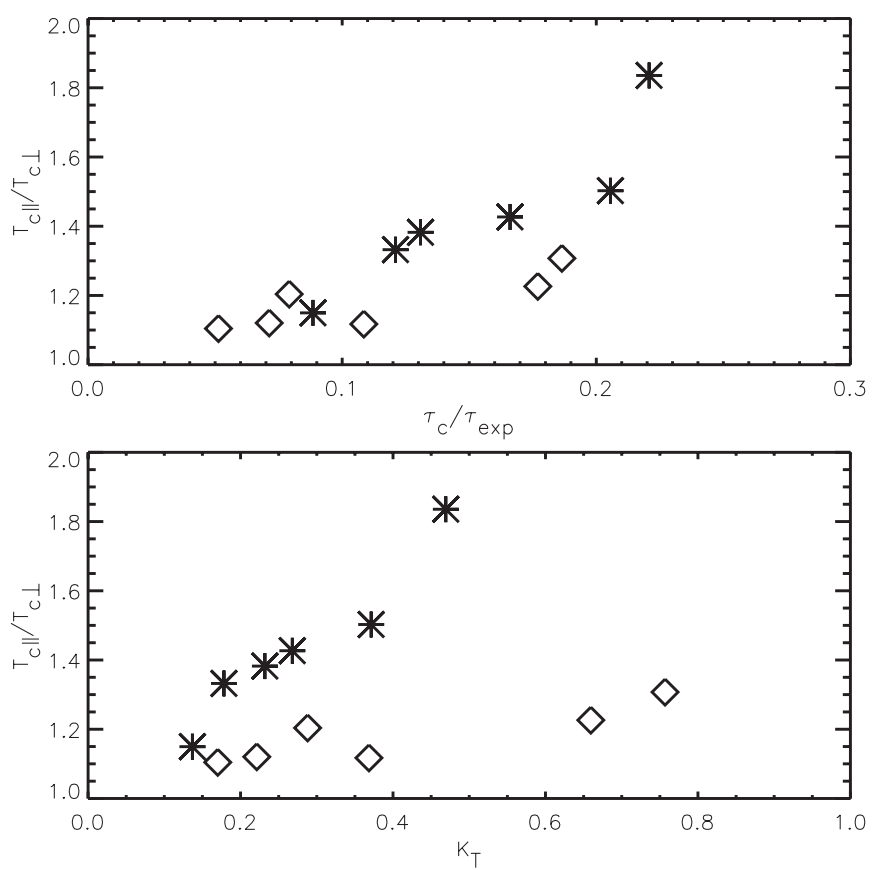

Figure 10. Same as Figure 9 for the cold component of the electron velocity distribution function.

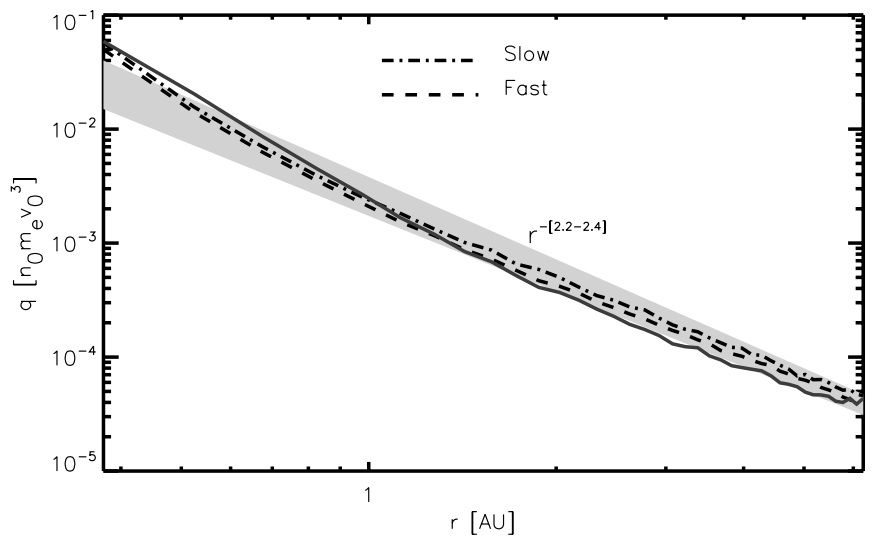

Figure 11. Electron heat flux vs. distance for a typical slow and fast wind (both cases have $n_{0}=27 \mathrm{~cm}^{-3}$ ). The shaded region outlines the asymptotic heat fluxes measured in all simulations, with logarithmic slopes in the range -2.2 and -2.4 . The solid line is the heat flux profile predicted by Equation (13) (see Feldman et al. 1975).

$L=T /|\nabla T|$ being the typical scale of variation of the temperature. In the bottom panels, the temperature anisotropies are plotted against the thermal Knudsen number $K_{\mathrm{T}}=\lambda_{\mathrm{c}} / L$ with $\lambda_{\mathrm{c}}$ being the local mean free path for a thermal electron. As already pointed out in Landi et al. (2010), we observe a good correlation between the temperature anisotropy and the collisional age as in solar wind observations (Salem et al. 2003; Štverák et al. 2008) regardless of whether we use the complete eVDF or only the cold component. The correlation is lost when we plot the temperature anisotropy against the Knudsen number where we observe that the anisotropy depends on the expansion velocity.

\subsection{Electron Heat Flux}

In Figure 11 the electron heat flux is plotted against the radial distance for two simulations, both having a base density of about $n_{0}=27 \mathrm{~cm}^{-3}$ and extending to $6 \mathrm{AU}$. For these two

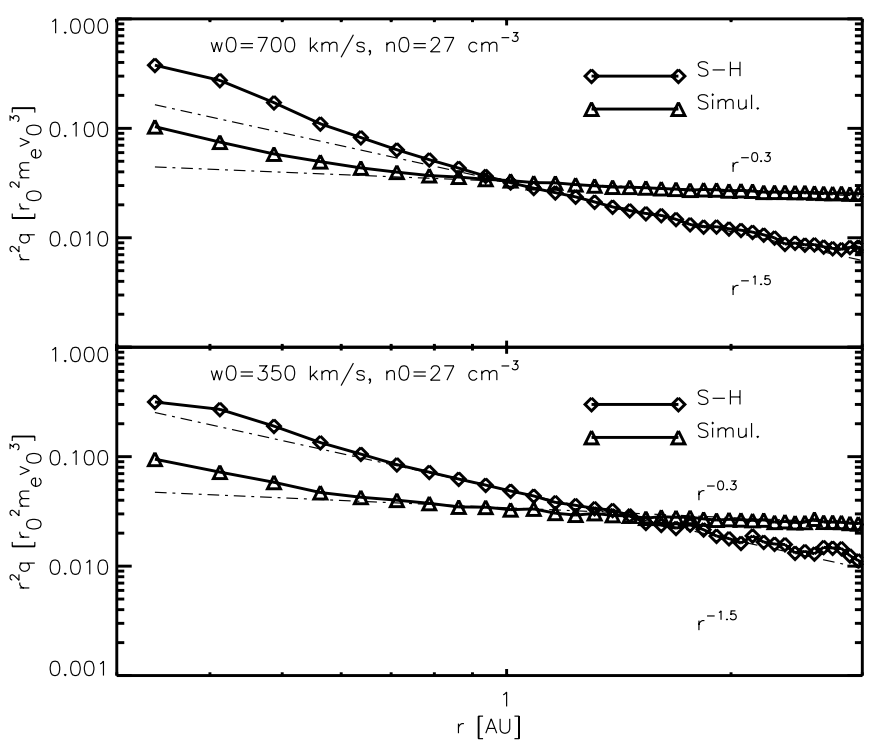

Figure 12. Heat flux profiles (triangles) scaled by $r^{2}$ for a fast (top) and slow (bottom) wind. Diamonds correspond to the collisional heat flux predicted by Equation (15). The dash-dotted lines highlight the asymptotic slopes for the curves reported here.

simulations, and more generally in all of our simulations, we find that the heat flux decreases approximately as a power law with an index in the range $[-2.3,-2.5]$ with steeper slopes for the more collisional winds. The slopes reveal that heating is provided by the electron thermal conduction although the slopes observed in the simulations are somewhat less steep than reported by Scime et al. (1994) using in-ecliptic Ulysses data in the range 1-5 $\mathrm{AU}$, where they observe a power-law index of about -3 . Similar results have been obtained with out-ofecliptic data (Scime et al. 1999) and data including the effect of solar cycle activity variations (Scime et al. 2001). In Figure 11, we have also plotted the bi-Maxwellian phenomenological heat flux model proposed by Feldman et al. (1975):

$$
q_{e} \simeq \frac{1}{2} n_{\mathrm{h}} w_{\mathrm{h}} k_{\mathrm{B}}\left[3\left(T_{\mathrm{h} \|}-T_{\mathrm{c} \|}\right)+2\left(T_{\mathrm{h} \perp}-T_{\mathrm{c} \perp}\right)\right] .
$$

The gray solid line refers to the data fitting obtained from the slow wind simulation reported in the same figure, showing an excellent agreement between the observed heat flux and the empirical formula equation (13). The good agreement between the measured flux and the empirical formula equation (13) implies that the electron heat flux is mainly due to the relative drift of the hot population with respect to the bulk motion of the whole plasma. A similar result has been obtained by Feldman et al. (1975) and Scime et al. (1994) for the real solar wind.

Not surprisingly, the electron heat flux cannot be conveniently described by the classical formula for a collisional-dominated plasma (Spitzer \& Härm 1953). In Figure 12 the heat-flux profiles scaled by $r^{2}$ for two simulations are reproduced, both having the same density but different velocities. In the same figure the equivalent collisional-dominated heat flux is also reproduced, given by (Braginskii 1965)

$$
\mathbf{q}_{\mathrm{sh}}=-3.19 \frac{p_{e} k_{\mathrm{B}}}{v_{\mathrm{ep}} m_{e}} \nabla T
$$

For purely radial variations of the temperature, the absolute value of the collisional flux can then be rewritten in terms of the 


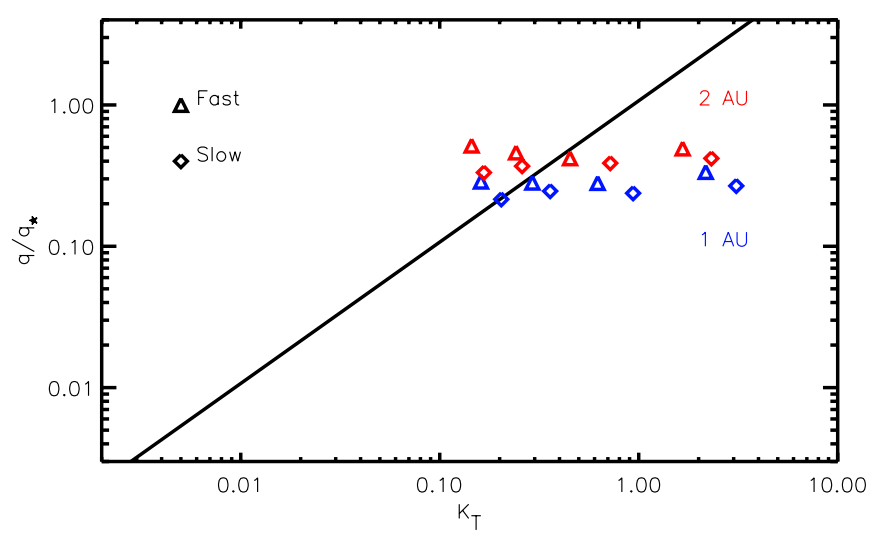

Figure 13. Normalized heat flux $q / q_{\star}$ (see Equation (16)) as a function of the thermal Knudsen number at 1 and $2 \mathrm{AU}$ for all simulations in Table 1 . The solid line corresponds to the normalized Spitzer-Härm flux (17).

(A color version of this figure is available in the online journal.)

thermal Knudsen number as

$$
q_{\mathrm{sh}}=\frac{3.19}{\sqrt{2}} p_{e} \sqrt{\frac{k_{\mathrm{B}} T}{m_{e}}} K_{\mathrm{T}} .
$$

From Figure 12, it is clear that both the slopes and the magnitude of the measured heat flux are completely different from that predicted by the classical formula: the collisional-dominated heat flux prediction appears to have a logarithmic slope of about -3.5 , consistent with a total electron temperature logarithmic slope in the range $[-0.8,-0.7]$, which is substantially steeper than observed in the solar wind. The Spitzer-Härm flux appears to be two to three times larger than the measured heat flux in the inner part of the simulation domain. At larger distances, because of its faster decrease, the measured heat flux outruns the Spitzer-Härm flux.

In Figure 13 the heat flux at $1 \mathrm{AU}$ and $2 \mathrm{AU}$ for all simulations are reported. The heat flux has been normalized to the freestreaming heat flux (Hundhausen 1972):

$$
q_{\star}=\frac{3}{2} n_{e} k_{\mathrm{B}} T_{e} v_{\| e},
$$

with $v_{\| e}^{2}=2 k_{\mathrm{B}} T_{\| e} / m_{e}$. The free-streaming flux $q_{\star}$ is essentially an upper limit for the flux as it corresponds to the case where the whole thermal energy per particle $1.5 k_{\mathrm{B}} T$ is advected at the electron thermal speed. With the flow being along the radial direction, the pertinent advection velocity is the parallel thermal velocity rather than the global thermal velocity. In the same figure, following Salem et al. (2003), we also report the normalized Spitzer-Härm flux:

$$
\frac{q_{\mathrm{sh}}}{q_{\star}}=\frac{3.19}{3} K_{\mathrm{T}} .
$$

From the figure we note that the normalized heat flux is almost independent of the Knudsen number and, at 1 AU we have $q_{e} / q_{\star} \simeq 0.3$, a value close to that observed by Salem et al. (2003). However, in our case the Spitzer-Härm heat flux does not appear to be an upper limit for the electron heat flux as for Salem et al. (2003). As already stated, in our simulations this is due to the fact that the measured heat flux decreases more slowly with distance than the Spitzer-Härm flux, the former becoming dominant beyond 1 AU (see Figure 12). This is also consistent with Scime et al. (1994) who observe electron

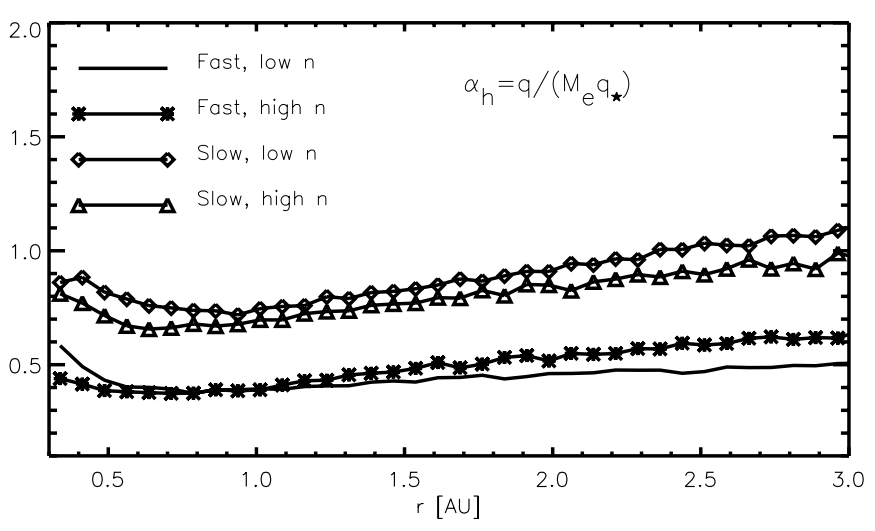

Figure 14. Radial evolution of the $\alpha_{\mathrm{h}}$ parameter from the collisionless heat flux expression (18) for various kinds of wind.

heat fluxes exceeding the Spitzer-Härm value in the solar wind beyond 2 AU.

Another interesting point which can be inferred from Figure 13, also observed by Salem et al. (2003), is the fact that the electron heat flux approaches and eventually exceeds the Spitzer-Härm heat flux at low Knudsen numbers. In our simulations, this occurs for $K_{\mathrm{T}} \lesssim 0.2$. This fact can be understood assuming a collisionless form of the heat flux inspired by the exospheric model proposed some time ago by Hollweg (1974):

$$
q_{\mathrm{nc}}=\alpha_{\mathrm{h}} \frac{3}{2} n_{e} k_{\mathrm{B}} T_{e} w_{0}=\alpha_{\mathrm{h}} q_{\star} M_{e}
$$

In this equation, $\alpha_{\mathrm{h}}$ is an order unity coefficient which depends on the electron temperature and bulk velocity, and $M_{e}=w_{0} / v_{\| e}$ is the electron Mach number. Using Equations (18) and (17) we can write the ratio of the two heat fluxes (Landi \& Pantellini 2003), viz.,

$$
\frac{q_{\mathrm{nc}}}{q_{\mathrm{sh}}}=\frac{3 \alpha_{\mathrm{h}}}{3.19} \frac{M_{e}}{K_{\mathrm{T}}} .
$$

From the equation it appears that the Spitzer-Härm flux exceeds the collisionless heat flux provided

$$
K_{\mathrm{T}}>\frac{3 \alpha_{\mathrm{h}}}{3.19} M_{e}
$$

In the inner domain of the simulation, for fast streams, $M_{e} \approx 0.3$ and using Equations (5)-(9) from Hollweg (1974) we have $\alpha_{\mathrm{h}} \approx 0.6$. According to Equation (20), $q_{\mathrm{sh}}>q_{\mathrm{nc}}$ if $K_{\mathrm{T}}>0.17$ (see also Hollweg 1976). For slow streams, $M_{\mathrm{e}} \approx 0.15, \alpha_{\mathrm{h}} \approx 1$, and $K_{\mathrm{T}}>0.14$. In the inner portion of the simulation domain $K_{\mathrm{T}}$ is always larger than 0.2 and the Spitzer-Härm flux effectively exceeds the collisionless flux.

Since $K_{\mathrm{T}} \propto T_{e}^{2} \nabla T_{e} /\left(n T_{e}\right)$, and $M_{e} \propto 1 / \sqrt{T_{e}}$, and assuming a power-law temperature $T \propto r^{-\beta_{\mathrm{e}}}$ one has $M_{e} / K_{\mathrm{T}} \propto r^{5 \beta_{e}-2}$. Thus, if $\beta_{e}>2 / 5=0.4$, which is the case in all simulations, and assuming a constant value for the coefficient $\alpha_{\mathrm{h}}$ (see below), the ratio $q_{\mathrm{nc}} / q_{\mathrm{sh}}$ must increase with distance and eventually exceed unity at some critical heliocentric distance that is shorter for fast streams as shown in Figure 12.

In Figure 14, the radial variation of the $\alpha_{\mathrm{h}}$ parameter from the collisionless expression for the flux (18) is plotted for fast and slow wind velocities. The most striking feature is that for the slow wind case, $\alpha_{\mathrm{h}}$ is roughly twice the fast wind case, indicating, as was already apparent in Figure 13, that $q / q_{*}=\alpha_{\mathrm{h}} M_{e}$ is less dependent on wind velocity than $\alpha$ itself. This also appears to be once more consistent with the Holwegg's 
expression where an anticorrelation between $\alpha_{\mathrm{h}}$ and the solar wind velocity is predicted (see Figure 3 in Hollweg 1974). The other interesting point is the overall tendency of $\alpha_{\mathrm{h}}$ to grow with distance, at least after a short decreasing phase near the lower boundary $r=r_{0}$. This tendency is also visible in Figure 13, indicating that both $\alpha_{\mathrm{h}} M_{e}$ and $\alpha_{\mathrm{h}}$ are growing functions of distance $r$. The overall conclusion is that a comparable value of $\alpha_{\mathrm{h}}$ may be used for fast and slow streams with a radial growth of the order of 0.1 per AU.

\section{DISCUSSION AND CONCLUSION}

We have presented stationary simulations of the solar wind using a fully kinetic, electron-proton, spherically symmetric code, which includes the effect of binary Coulomb collisions and where the charge neutralizing electric field is computed self-consistently. A purely radial magnetic field is assumed. The simulation domain covers a heliocentric distance in the range $0.3 \mathrm{AU}$ to 3 or $6 \mathrm{AU}$, where the wind has already reached its asymptotic velocity and where the effect of gravity can safely be neglected. In our study, we have focused primarily on the evolution of the eVDF and its moments from the base of the simulation domain at $r=r_{0}$ where we assume an isotropic Maxwellian eVDF. As we move to larger values of $r$, we observe the formation of an eVDF with a slightly anisotropic collision-dominated core, and a radially antisunward drifting hot beam population. This collimated beam, made of electrons with speeds exceeding a few times $(\sim 4)$ the electron thermal velocity, corresponds to the extrathermal population predicted by Scudder \& Olbert (1979). The relative drift velocities and the densities of the two populations are compatible with the core-strahl properties observed in the solar wind.

The electron temperature appears to decrease with the distance with logarithmic slopes in the range 0.6-0.9 in rather good agreement with solar wind observations. Moreover, as noted by Cranmer et al. (2009) for the real wind, we observe a flattening of the temperature profile at larger distances and show that the flattening is mainly due to the quasi-isothermal behavior of the hot component as already observed in exospheric wind models (Meyer-Vernet \& Issautier 1998). The electron temperature anisotropy of the cold population in our simulations is found to be in good agreement with those observed for the core component in the solar wind (Štverák et al. 2008). On the other hand, the total electron temperature anisotropy is larger than the anisotropy measured at 1 AU by Salem et al. (2003). Several reasons can account for the difference. (1) Coulomb collisions are not efficient enough to scatter high-energy electrons to large pitch angles (also see Pierrard et al. 2001a). (2) Particle scattering by sunward propagating whistler waves (Vocks et al. 2005; Saito \& Gary 2007a, 2007b) and/or the instability of the beam component itself (Gary et al. 1994) may play a role in reducing the anisotropy in the real wind but are not included in our simulations. (3) Magnetic field spiraling due to the Sun's rotation is not included in our simulations. Spiraling forces the close to ecliptic magnetic field intensity to decrease more slowly with distance than in a radial field keeping the perpendicular temperature at a higher level.

The electron heat flux measured in our simulations drops faster than $r^{-2}$ implying a deposition of heat in the plasma and a non-adiabatic temperature profile. The decrease of the heat flux with distance appears to be less steep in our simulations compared to the solar wind (Scime et al. 1994, 1999, 2001). The difference could be related to the presence of electromagnetic heat flux instabilities (Gary et al. 1994) as well as scattering due to turbulent fluctuations at electronic scales. As shown by Feldman et al. (1975) for the case of the solar wind, confirmed by our simulations, the heat flux is mainly carried by the antisunward drifting magnetic field aligned beam. As expected, given the relatively high value of the Knudsen number, the collisional expression for the heat flux (Spitzer \& Härm 1953) is unable to provide a convenient estimate of the flux. A better estimate for the radial electron energy flux in our simulations is provided by the collisionless model first proposed by Hollweg (1974).

Despite its obvious shortcomings, our model has been able to capture many properties of the solar wind electrons from various interplanetary probes, using different instruments and techniques. The fact that no such things as turbulence, reconnection, wave-particle interactions, shocks, etc., are included in our simulations suggests that turbulent dissipation at electronic scales may not be easily estimated using the present data. On the other hand, Coulomb collisions appear to be an essential ingredient in shaping the eVDF, at least beyond the region of acceleration. Thus, any reasonable kinetic model of the solar wind expansion has to include collisions to conveniently describe the dynamics of the eVDF.

The research leading to these results has received funding from the European Commission's Seventh Framework Programme (FP7/2007-2013) under the grant agreement SHOCK (project number 284515).

\section{REFERENCES}

Braginskii, S. I. 1965, in Reviews of Plasma Physics, Vol. 1, ed. M. A. Leontovich (New York: Consultants Bureau), 205

Chen, Y., Esser, R., \& Hu, Y. Q. 2003, J. Geophys. Res. (Space Phys.), 108,1371

Chew, G. F., Goldberger, M. L., \& Low, F. E. 1956, Proc. R. Soc. A, 236, 112

Cranmer, S. R., Matthaeus, W. H., Breech, B. A., \& Kasper, J. C. 2009, ApJ, 702, 1604

Cuperman, S., Detman, T., \& Dryer, M. 1988, ApJ, 330, 466

Feldman, W. C., Anderson, R. C., Asbridge, J. R., et al. 1982, J. Geophys. Res., 87,632

Feldman, W. C., Asbridge, J. R., Bame, S. J., Gary, S. P., \& Montgomery, M. D. 1976a, J. Geophys. Res., 81, 2377

Feldman, W. C., Asbridge, J. R., Bame, S. J., Gosling, J. T., \& Lemons, D. S. 1978a, J. Geophys. Res., 83, 5285

Feldman, W. C., Asbridge, J. R., Bame, S. J., Gosling, J. T., \& Lemons, D. S. 1978b, J. Geophys. Res., 83, 5297

Feldman, W. C., Asbridge, J. R., Bame, S. J., Gosling, J. T., \& Lemons, D. S. 1979, J. Geophys. Res., 84, 4463

Feldman, W. C., Asbridge, J. R., Bame, S. J., Montgomery, M. D., \& Gary, S. P. 1975, J. Geophys. Res., 80, 4181

Feldman, W. C., Asbridge, J. R., Bame, S. J., et al. 1976b, J. Geophys. Res., 81,5207

Gary, S. P., Scime, E. E., Phillips, J. L., \& Feldman, W. C. 1994, J. Geophys. Res., 99, 23391

Hartle, R. E., \& Sturrock, P. A. 1968, ApJ, 151, 1155

Hollweg, J. V. 1974, J. Geophys. Res., 79, 3845

Hollweg, J. V. 1976, J. Geophys. Res., 81, 1649

Hundhausen, A. J. 1972, Coronal Expansion and Solar Wind (Berlin: Springer)

Issautier, K., Meyer-Vernet, N., Moncuquet, M., \& Hoang, S. 1998, J. Geophys. Res., 103, 1969

Jockers, K. 1970, A\&A, 6, 219

Lamy, H., Pierrard, V., Maksimovic, M., \& Lemaire, J. F. 2003, J. Geophys. Res. (Space Phys.), 108, 1047

Landi, S., \& Pantellini, F. 2003, A\&A, 400, 769

Landi, S., Pantellini, F., \& Matteini, L. 2010, in AIP Conf. Ser., Vol. 1216, Twelfth International Solar Wind Conference, ed. M. Maksimovic, K. Issautier, N. Meyer-Vernet, M. Moncuquet, \& F. Pantellini (Melville, NY: AIP), 218

Landi, S., \& Pantellini, F. G. E. 2001, A\&A, 372, 686

Le Chat, G., Issautier, K., Meyer-Vernet, N., \& Hoang, S. 2011, Sol. Phys., 271, 141 
Lemaire, J., \& Scherer, M. 1971, J. Geophys. Res., 76, 7479

Lie-Svendsen, Ø., Hansteen, V. H., \& Leer, E. 1997, J. Geophys. Res., 102,4701

Lie-Svendsen, Ø., \& Leer, E. 2000, J. Geophys. Res., 105, 35

Maksimovic, M., Gary, S. P., \& Skoug, R. M. 2000, J. Geophys. Res., 105,18337

Maksimovic, M., Pierrard, V., \& Lemaire, J. F. 1997a, A\&A, 324, 725

Maksimovic, M., Pierrard, V., \& Riley, P. 1997b, Geophys. Res. Lett., 24, 1151

Maksimovic, M., Zouganelis, I., Chaufray, J.-Y., et al. 2005, J. Geophys. Res. (Space Phys.), 110, A09104

Marquardt, D. W. 1963, J. Soc. Indust. Appl. Math., 11, 431

Marsch, E., Thieme, K. M., Rosenbauer, H., \& Pilipp, W. G. 1989, J. Geophys. Res., 94, 6893

Matteini, L., Landi, S., Hellinger, P., et al. 2007, Geophys. Res. Lett., 34, 20105

Meyer-Vernet, N., \& Issautier, K. 1998, J. Geophys. Res., 103, 29705

Montgomery, M. D., Bame, S. J., \& Hundhausen, A. J. 1968, J. Geophys. Res., 73,4999

Ogilvie, K. W., \& Scudder, J. D. 1978, J. Geophys. Res., 83, 3776

Pantellini, F., \& Landi, S. 2001, Ap\&SS, 277, 149

Pantellini, F. G. E. 2000, Am. J. Phys., 68, 61

Phillips, J. L., Bame, S. J., Gary, S. P., et al. 1995a, Space Sci. Rev., 72, 109

Phillips, J. L., Feldman, W. C., Gosling, J. T., \& Scime, E. E. 1995b, Adv. Space Res., 16, 95

Phillips, J. L., \& Gosling, J. T. 1990, J. Geophys. Res., 95, 4217

Phillips, J. L., Gosling, J. T., McComas, D. J., et al. 1989, J. Geophys. Res., 94, 6563

Pierrard, V., Issautier, K., Meyer-Vernet, N., \& Lemaire, J. 2001a, Geophys. Res. Lett., 28, 223

Pierrard, V., Maksimovic, M., \& Lemaire, J. 1999, J. Geophys. Res., 104, 17021

Pierrard, V., Maksimovic, M., \& Lemaire, J. 2001b, J. Geophys. Res., 106,29305
Pilipp, W. G., Muehlhaeuser, K.-H., Miggenrieder, H., Montgomery, M. D., \& Rosenbauer, H. 1987, J. Geophys. Res., 92, 1075

Pilipp, W. G., Muehlhaeuser, K.-H., Miggenrieder, H., Rosenbauer, H., \& Schwenn, R. 1990, J. Geophys. Res., 95, 6305

Rosenbauer, H., Schwenn, R., Marsch, E., et al. 1977, J. Geophys. Z. Geophys., 42,561

Saito, S., \& Gary, S. P. 2007a, Geophys. Res. Lett., 34, 1102

Saito, S., \& Gary, S. P. 2007b, J. Geophys. Res., 112, 6116

Salem, C., Hubert, D., Lacombe, C., et al. 2003, ApJ, 585, 1147

Schulz, M., \& Eviatar, A. 1973, J. Geophys. Res., 78, 3948

Schwenn, R. 1990, Large-Scale Structure of the Interplanetary Medium, Vol. 1 (1st ed.; Berlin: Springer), 99

Scime, E. E., Badeau, A. E., \& Littleton, J. E. 1999, Geophys. Res. Lett., 26, 2129

Scime, E. E., Bame, S. J., Feldman, W. C., et al. 1994, J. Geophys. Res., 99, 23401

Scime, E. E., Littleton, J. E., Gary, S. P., Skoug, R., \& Lin, N. 2001, Geophys. Res. Lett., 28, 2169

Scudder, J. D., \& Olbert, S. 1979, J. Geophys. Res., 84, 2755

Sittler, E. C., Jr., \& Scudder, J. D. 1980, J. Geophys. Res., 85, 5131

Sittler, E. C., Jr., Scudder, J. D., \& Jessen, J. 1981, in Solar Wind 4 Conference, ed. H. Rosenbauer (Katlenburg-Lindau: Max-Planck Institute für Aeronomie), 257

Smith, H. M., Marsch, E., \& Helander, P. 2012, ApJ, 753, 31

Spitzer, L., \& Härm, R. 1953, Phys. Rev., 89, 977

Štverák, Š., Maksimovic, M., Trávníček, P. M., et al. 2009, J. Geophys. Res. (Space Phys.), 114, 5104

Štverák, Š., Trávníček, P., Maksimovic, M., et al. 2008, J. Geophys. Res. (Space Phys.), 113, 3103

Vocks, C., Salem, C., Lin, R. P., \& Mann, G. 2005, ApJ, 627, 540

Zouganelis, I., Maksimovic, M., Meyer-Vernet, N., Lamy, H., \& Issautier, K. 2004, ApJ, 606, 542

Zouganelis, I., Meyer-Vernet, N., Landi, S., Maksimovic, M., \& Pantellini, F. 2005, ApJ, 626, L117 